\title{
TRATAMENTO CIRÚRGICO DA LITÍASE VESICULAR NO IDOSO: ANÁLISE DOS RESULTADOS IMEDIATOS DA COLECISTECTOMIA POR VIA ABERTA E VIDEOLAPAROSCÓPICA
}

Ronaldo elias Carnut Rêgo*, Tércio de Campos, André de Moricz, Rodrigo Altenfelder Silva, Adhemar Monteiro Pacheco Júnior

Trabalho realizado no Grupo de Vias Biliares e Pâncreas do Departamento de Cirurgia da Faculdade de Ciências Médicas da Santa Casa de São Paulo, SP.

RESUMO - 0 aumento da expectativa de vida faz com que os idosos sejam a faixa etária com maior crescimento. A colecistectomia é a cirurgia abdominal mais comum neste grupo. $A$ associação de doenças crônicas e formas complicadas implica em preconizar 0 tratamento operatório eletivo nos idosos sintomáticos. Os bons resultados obtidos com a colecistectomia laparoscópica determinaram o surgimento de estudos comparando a cirurgia convencional com a videocirurgia, entretanto, nos idosos há poucas casuísticas analisadas, principalmente no nosso meio.

Objetivos. Analisar os resultados imediatos da morbidade da colecistectomia eletiva em doentes idosos, por duas vias de acesso.

Métodos. Estudamos, retrospectivamente, doentes com idade $\geq 65$ anos operados por incisão subcostal (grupo l) e videolaparoscópica (grupo II). Os grupos foram comparados em relação à presença de complicação até 30 dias e nas suas formas de apresentação: cirúrgica, cirúrgicas (intra e pós-operatórias) e sistêmicas e, posteriormente, com as variáveis sexo, faixa etária, doença associada, cardiovascular, metabólica, pulmonar e ASA. Na análise estatística, empregou-se $o$ teste $t$ de Student e a correlação de Spearman, considerando $p \leq 0,05$ como significância estatística.

Resultados. Foram 246 doentes distribuídos em dois grupos, sendo I 20 no grupo I e 126 no grupo II. A média de idade foi de 7 I anos em ambos os grupos. As doenças associadas estiveram presentes em 155 doentes (63\%), destacando-se as cardiovasculares, com distribuição semelhante nos grupos I e II. A conversão ocorreu em 10 doentes $(8,3 \%)$. 0 tempo cirúrgico médio foi de 133 minutos no grupo I e de II 2 minutos no grupo II $(p=0.00 I)$. A permanência hospitalar em dias foi semelhante entre os grupos I $(3,3)$ e II $(3,2)$ $p=0,698$. A morbidade foi semelhante nos grupos I $(10,8 \%)$ e II $(10,3 \%), p=0,896$. Não houve diferença estatística em relação à presença de complicação e de suas formas de apresentação entre os grupos. A presença de complicação pós-operatória no sexo feminino no grupo II $(\mathrm{p}=0,026)$ foi a única variável com significância estatística. A mortalidade ocorreu em dois doentes $1,6 \%$ ambos do grupo II $(p=0,158)$.

Conclusóes. 0 doente idoso portador de litíase vesicular sintomática e clinicamente compensado pode ser submetido à colecistectomia de forma eletiva, com baixos índices de morbimortalidade e resultados semelhantes, independente da via de acesso empregada.

UnITERMos: Colecistectomia. Laparoscopia. Idoso. Complicação.

\section{INTRODUÇÃO}

A população de idosos é a que apresenta a maior projeção de crescimento mundial. No Brasil, estima-se o aumento dos idosos de 7,5 para 30 milhões em 2020, ocasião na qual o país terá a sexta população de idosos do planeta'.

Em decorrência das características específicas dos países de clima tropical, considera-se idoso o indivíduo acima de 60 anos de idade 2 . Entretanto, na literatura internacional, a idade de 65 anos é mais aceita ${ }^{3-14}$.

\footnotetext{
* Correspondência:

Rua Cesário Mota Júnior, 112

Departamento de Cirurgia

01221-020 - São Paulo - SP

cir.pancreas@santacasasp.org.br
}

A diminuição das reservas funcionais e a presença de doenças associadas em cerca da metade dessa população estabelecem um grupo de doentes com maior complexidade clínica. Concomitante às doenças crônicas, algumas enfermidades de tratamento cirúrgico apresentam aumento de incidência com a progressão da idade ${ }^{15}$.

A colelitíase éa doença cirúrgica abdominal mais comum no doente idoso, sendo sua incidência relacionada à progressão da idade, com prevalência global de $9,3 \%$; passando para $21,4 \%$ nos idosos de 60 a 69 e, na faixa etária acima de 70 anos, acomete $27,5 \%$ dos indivíduos ${ }^{16}$. As colecistectomias atualmente realizadas nessa população variam de $8,3 \%$ a $24 \% 6,7,12,13,18$. E, com publicações enfatizando 0 aumento de cirurgias em octagenários ${ }^{19-21}$.
A colelitíase apresenta características próprias na população idosa, como a aproximação da incidência entre os $\operatorname{sexos}^{22}$, a coledocolítiase é mais freqüente ${ }^{19}$, há uma maior prevalência de câncer da vesícula biliar $^{22}$. Comumente, a doença calculosa biliar é assintomática ${ }^{19} \mathrm{e}$ um aspecto de extrema importância é a sua maneira de manifestação, sendo freqüente a presença de quadros de agudizaçãa $0^{6} \mathrm{e}$ formas complicadas da doença" vezes a mortalidade na cirurgia biliar de urgência, quando comparada com a cirurgia eletiva ${ }^{19,23}$.

Desta forma, estes doentes devem ter indicação cirúrgica preferencialmente eletiva, com adequado preparo pré-operatório para diminuir os riscos do procedimento ${ }^{24}$. 
RêGo REC ET AL.

Ao final dos anos 80 , a colecistectomia videolaparoscópica iniciou uma verdadeira revolução na cirurgia abdominal. Essa nova técnica difundiu-se por toda a comunidade cirúrgica mundial de forma muito rápida ${ }^{25,26}$, sendo introduzida no Brasil a partir de $1990^{27}$. Demonstrou vantagens: menor trauma cirúrgico, menor tempo de internação, retorno precoce às atividades profissionais e melhor resultado estético. Suplantou contraindicações como obesidade, coagulopatia, doença pulmonar crônica obstrutiva, cirurgia prévia e colecistite agudaa ${ }^{26,28}$. Na cirurgia convencional, destacam-se as complicações clínicas, principalmente cardiopulmonares e as relacionadas às incisões abdominais ${ }^{29}$. Entretanto, apesar dos seus benefícios, a videocirurgia demonstrou complicações habitualmente menos freqüentes na cirurgia convencional, como fístulas biliares, hemorragias, iatrogenias relacionadas à realização do pneumoperitônio e principalmenteà temida e muitas vezes complexa lesão da via biliar princi$\mathrm{pal}^{30}$. Todavia, a segurança adquirida com a colecistectomia laparoscópica permitiu a inclusão de doentes idosos e, a partir de 1993, encontram-se publicações analisando a videocirurgia neste grupo de doentes $3,4,6,6,9,10-13,18,20,31$ com algumas comparando a morbidade entre os dois métodos ${ }^{5,8,14,21,32}$.

Analisando na literatura a população idosa, observamos, no grupo operado por laparoscopia, aspectos semelhantes na morbidade com relação aos trabalhos que não avaliam exclusivamente esta população, ou seja, maior número de complicações cirúrgi$\operatorname{cas}^{11,12}$ e menor número de complicações cardiopulmonares ${ }^{8,31}$.

No Brasil, há poucas publicações acerca da colecistectomia por via laparoscópica em idosos ${ }^{6}$ e não observamos artigos comparando os dois métodos nesta população.

Desse modo, o presente estudo tem por objetivo estudar os resultados imediatos da colecistectomia eletiva em doentes idosos sintomáticos operados por incisão subcostal evideolaparoscópica.

\section{Métodos}

Estudamos, retrospectivamente, doentes idosos com diagnóstico de colelitíase, submetidos à colecistectomia eletiva no Departamento de Cirurgia da Faculdade de Ciências
Médicas da Santa Casa de São Paulo, no período de abril de 1992 a março de 1999. Selecionamos doentes com idade mínima de 65 anos, portadores de litíase vesicular, confirmada por exame ultrassonográfico, clinicamente sintomáticos e compensados de suas doenças associadas quando presentes. Neste período, 1708 doentes foram submetidos a colecistectomia. Destes, selecionamos 246 doentes com idade mínima de 65 anos, que correspondem a $\mid 4,4 \%$ da amostra. Duzentos doentes $(81,3 \%)$ pertenciam ao sexo feminino e $46(18,7 \%)$ ao masculino. A idade variou de 65 a 97 anos, com média de 7l anos. Co-morbidades estiveram presente em 155 doentes (63\%) e a doença cardiovascular foi a mais freqüente. Os doentes foram classificados como ASA I a $3^{32}$.

Constituiu-se dois grupos, segundo avia de acesso utilizada: o grupo I com I 20 doentes submetidosàlaparotomia porvia aberta (incisão subcostal direita), conforme técnica padronizada no serviço (incisão sobre o reto abdominal), operados por residentes do segundo ano do programa de residência em cirurgia geral; o grupo Il com 126 doentes operados através de cirurgia videolaparoscópica, por residentes do terceiro equarto anos do programa de residência em cirurgia do aparelho digestivo. A colangiografia intra-operatória ea drenagem da cavidade foram realizadas de forma seletiva. A antibioticoprofilaxia foi realizada com cefalosporina de primeira geração.

Os grupos l ell foram divididos de acordo com as variáveis: média de idade, faixa etária (65 a 74 anos, e igual ou superior a 75 anos), sexo, presença de doença associada (cardiovascular, metabólica, pulmonar) e ASA I a 3.

Em relação ao tratamento operatório, estudamos os eventos intra-operatórios: frequêencias de colangiografia intra-operatória, abertura da vesícula, drenagem da cavidade, conversão da via de acesso cirúrgico (somente nos doentes do grupo II) e tempo operatório em minutos. Considerou-se a permanência hospitalar pós-operatória em dias.

Com relação ao período pós-operatório, os doentes foram classificados segundo a presença ou ausência de complicações precoces (até 30 dias), divididas em cirúrgicas, cirúrgicas intra-operatórias e, pós-operatórias, e sistêmicas. Inicialmente comparadas entre os grupos e posteriormente analisadas em relação às variáveis pré-operatórias.
A mortalidade foi analisada de forma descritiva.

A análise estatística foi realizada através da correlação de Spearman para as variáveis contínuas e pelo teste tde Student para as variáveis numéricas, observando-se níveis de significância iguais ou inferiores a $5 \%$.

\section{Resultados}

Comparando-se a presença das variáveis: média de idade, faixa etária, sexo, doença associada e de suas formas cardiovascular (DCV), metabólica (DM), pulmonar (DP), e ASA (I, 2 e 3) observamos distribuição semeIhante entre os grupos, não havendo diferença estatisticamente significante.

Em relação ao tratamento operatório, observamos que os eventos intra-operatórios: colangiografia grupo I ( $3 \mathrm{I} / \mathrm{I} 20$ doentes) e grupo II (23/I 26 doentes); abertura da vesícula grupo I (I7/I 20 doentes) e grupo II (35/I26 doentes) e drenagem da cavidade grupo I (3 I/ 120 doentes) e grupo II (66/126 doentes) foram estatisticamente significantes para as variáveis abertura de vesícula $(p=0,009)$ e drenagem da cavidade $(p<0,00 \mathrm{I})$, sendo mais freqüentes no grupo Il.

A conversão da via laparoscópica para laparotomia ocorreu em 10 doentes (8,3\%), entre os quais sete apresentaram aderências, dificultando a dissecção das estruturas do pedículo biliar. Houve falha na realização do pneumoperitônio em dois doentes e o décimo apresentou acidente de punção com lesão de alça de intestino delgado.

O tempo operatório apresentou diferença estatisticamente significante entre os grupos estudados sendo a média de 133 minutos no grupo I e de $1 \mid 2$ minutos no grupo II $(p=0,00 I)$.

A permanência hospitalar foi semelhante entre os dois grupos sendo de 3,3 dias no grupo l e 3,02 dias no grupo Il.

A taxa global de complicações foi de 10,6\% (26 doentes). Houve predomínio das complicações cirúrgicas em 20 doentes, sendo intra-operatórias em seis e pós-operatórias em catorze. As complicações sistêmicas ocorreram em quatro doentes e dois apresentaram complicações cirúrgicas e sistêmicas.

Nos doentes do grupo I, treze (10,8\%) apresentaram complicações com distribuição semelhante entre as três formas. As complicações cirúrgicas estiveram presentes em oito 


\begin{tabular}{|c|c|c|c|}
\hline \multicolumn{4}{|c|}{ Tabela I - Complicações encontradas no grupo I } \\
\hline \multirow[t]{2}{*}{ Doente } & \multicolumn{2}{|c|}{ Complicações cirúrgicas } & \multirow[t]{2}{*}{ Complicações sistêmicas } \\
\hline & Intra-operatória & Pós-operatória & \\
\hline I & lesão davia biliar principal & & \\
\hline 2 & lesãodeartériahepática & & \\
\hline 3 & & & atelectasia \\
\hline 4 & & infecçãodeparede & \\
\hline 5 & & Evisceração & \\
\hline 6 & & Infecção de parede & \\
\hline 7 & lesãohepática & & depressão respiratória \\
\hline 8 & & & IAM \\
\hline 9 & lesãode ramo portal & & \\
\hline 10 & & Evisceração & \\
\hline$\|$ & & Evisceração & \\
\hline 12 & & & atelectasia \\
\hline 13 & & & $\mathrm{AVCl}$ \\
\hline
\end{tabular}

\begin{tabular}{|c|c|c|c|}
\hline \multirow[t]{2}{*}{ Doente } & \multicolumn{2}{|c|}{ Complicações cirúrgicas } & \multirow[t]{2}{*}{ Complicações sistêmicas } \\
\hline & $\begin{array}{l}\text { Intra-operatória } \\
\text { enfisemadesubcutâneo }\end{array}$ & Pós-operatória & \\
\hline 2 & & migraçãododreno & \\
\hline 3 & & migraç̃o do dreno & \\
\hline 4 & & infecçãodeparede & \\
\hline 5 & & migraçãododreno & \\
\hline $6^{*}$ & lesãode intestino delgado & & \\
\hline 7 & lesãodeepíplon & & \\
\hline 8 & & fístulabiliar & \\
\hline $9 \bumpeq$ & & fístulabiliar & \\
\hline $10 \%$ & & coleção/evisceração & $\mathrm{AVCl} / \mathrm{BCP}$ \\
\hline $1 \|^{\beta}$ & & pancreatiteaguda & \\
\hline 12 & & migração do dreno & \\
\hline 13 & & fístulabiliar & \\
\hline
\end{tabular}

*conversão

$\Omega_{\text {neoplasia }}$

ఛóbito

\begin{tabular}{|c|c|c|}
\hline Complicação & $\begin{array}{c}\text { Grupo I* } \\
n^{\circ}(\%)\end{array}$ & $\begin{array}{c}\text { Grupo II* } \\
n^{0}(\%)\end{array}$ \\
\hline Complicação presenteausente & $13(11) 107(89)$ & $13(10) \mid 13(90)$ \\
\hline Cirúrgica presenteausente & $9(7,5)|I|(92,5)$ & $13(10) \mid 13(90)$ \\
\hline Intra-operatória presenteausente & $4(3) 116(97)$ & $3(2) 123(98)$ \\
\hline Pós-operatória presenteausente & $5(4) / 15(96)$ & $10(8) 116(92)$ \\
\hline Sistêmica presenteausente & $5(4) \mid 115(96)$ & $I(I) \mid 25(99)$ \\
\hline
\end{tabular}

${ }^{*} p>0,05$

doentes: três intra-operatórias e cinco pósoperatórias. As complicações sistêmicas estiveram presentes em quatro doentes. Um deles apresentou complicação cirúrgica intraoperatória esistêmica(Tabela I).
No grupo II, as complicações também estiveram presentes em I 3 doentes ( $10,3 \%$ ). Houve predomínio das complicações cirúrgicas presentes em 12 e, destes, três apresentaram complicações intra-operatórias e nove pós-operatórias. Um doente apresentou os dois tipos de complicações (cirúrgica pós-operatória e sistêmica) (Tabela 2).

Quando analisamos a ocorrência de complicações e estas nas suas formas de apresentação, observamos que não houve diferença estatisticamente significante entre os dois grupos (Tabela 3).

Os resultados entre as variáveis pré-operatórias analisadas e a ocorrência de complicações e, estas nas suas diversas formas, estão apresentadas na Tabela 4, observando-se que apenas as complicações pós-operatórias foram significativamente maiores nos doentes do sexo feminino, do grupo II $(p=0,026)$.

A mortalidade global foi de $0,8 \%$. Dois doentes do grupo II (I,6\%) do sexo masculino e ambos ASA 2. O resultado obtido na comparação do número de mortos entre os grupos não foi significativo $(p=0,158)$.

\section{Discussão}

Àsemelhança da colecistectomia convenciona ${ }^{24}$, é demonstrado que o método videolaparoscópico pode ser realizado por cirurgiões em formação, pois os resultados não diferem dos obtidos por cirurgióes experientes $^{33}$. Em nosso serviço, a preocupação com a formação de cirurgiões e a necessidade de tratar um maior número de doentes faz com que sejam mantidas as colecistectomias realizadas por via convencional evideolaparoscópica em proporções semelhantes e de forma aleatória.

Em nosso estudo, consideramos idoso os indivíduos acima de 65 anos e, em estudo prévio realizado no serviço, identificamos maior morbidade nos indivíduos acima de 75 anos operados eletivamente de litíase biliar ${ }^{34}$, justificando a divisão em faixas etárias.

A divisão por sexo assume aspectos particulares quando estudamos litíase vesicular, observando-se aproximação da incidência da doença entre os sexos com a progressão da idade ${ }^{22}$. No presente estudo, este aspecto não foi observado.

As doenças associadas, mais freqüentes nos idosos, relacionam-se à maior morbidade nestes doentes. Classicamente, são consideradas como fatores de risco e, em nosso estudo, apresentaram distribuição semelhante à observada na literatura, com predomínio das doenças cardiovasculares $s^{6,8,12,31,35}$. 
RêGo REC ET AL.

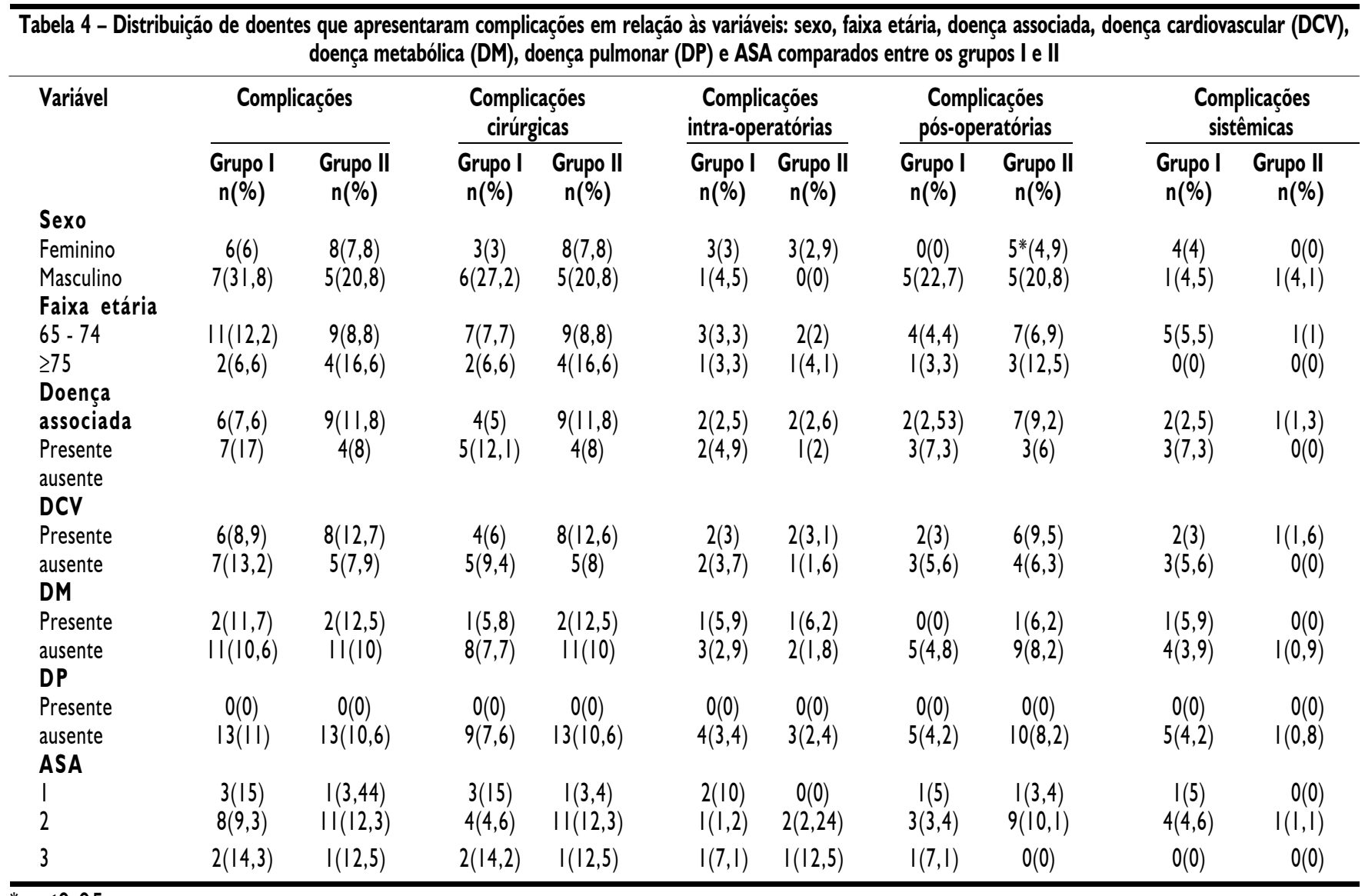

A classificação do estado físico da Associação Americana de Anestesiologia ${ }^{32}$ tem por objetivo avaliar o risco anestésico dos doentes, permitindo classificá-los em vários níveis e correlacioná-los com a morbidade encontrada. Neste estudo, os doentes classificaram-se entre os três primeiros graus, incluindo doenças graves clinicamente compensadas no préoperatório.

Observando a nossa casuística, encontramos características pré-operatórias semelhantes entre os doentes dos dois grupos, possibilitando a comparação entre os métodos.

Apesar da colecistectomia laparoscópica apresentar alguns aspectos próprios, o método não difere da cirurgia convencional em outros, um desses é com relação à colangiografia intra-operatória ${ }^{36,37}$ que é realizada de forma seletiva no nosso serviço, não havendo diferença estatisticamente significante nas taxas de execução nos dois grupos.

A colecistectomia laparoscópica acompanha-se de maior número de perfurações da vesícula biliar, sendo atribuída a aspectos téc- nicos com incidência variando de $0 \%$ a $30 \%^{38}$. Esta maior taxa, associada ao receio de escape das estruturas "clipadas," implicam no maior número de drenagem da cavidade abdomina ${ }^{39}$. Em nosso estudo, estes aspectos foram observados, sendo tanto a perfuração da vesícula biliar como a drenagem da cavidade abdominal mais freqüentes no grupo II, com diferença estatisticamente significante.

A conversão é uma particularidade da videolaparoscopia e diversos são os fatores determinantes, destacando-se a dificuldade técnica na dissecção das estruturas do pedículo biliar, que em idosos é a principal causa de conversão. Areincidência de surtos inflamatórios nesse grupo de doentes determina acentuada alteração anatômica e contribui para a conversão do método em cerca de $10 \%$ nos procedimentos eletivos ${ }^{10,12,40}$. Um aspecto a ser considerado é quanto à ocorrência de complicações em doentes com procedimento convertido para laparotomia. Adotando-se o princípio da intenção de tratar, a morbidade encontrada nesses doentes é atribuída ao mé- todo inicial de tratamento ${ }^{41}$. A conversão, em nossa amostra, foi realizada em 10 doentes (8.3\%), compatível com a literatura.

$\mathrm{O}$ acesso à cavidade peritoneal pela videocirurgia aboliu o tempo despendido com a diérese e síntese da parede abdominal. Entretanto, um estudo multicêntrico prospectivo com 359 doentes operados por laparoscopia, demostrou que $34 \%$ do tempo do procedimento foi relacionado à passagem e retirada dos trocartes ${ }^{42}$. Outros referiram tempo cirúrgico maior na colecistectomia laparoscópica $^{43,44}$. Há ainda estudos com doentes idosos, em que o tempo cirúrgico foi semelhante ou pouco maior na cirurgia convenciona ${ }^{8,14,45}$.

Diversos são os fatores determinantes do tempo operatório, sendo a experiência do cirurgião um dos principais ${ }^{33,42}$. Neste trabalho, o maior tempo cirúrgico observado no grupo I esteve possivelmente relacionado às características próprias do nosso serviço, onde as cirurgias convencionais são preferencialmente realizadas pelos residentes do segundo ano do programa de cirurgia geral e as 
colecistectomias laparoscópicas por residentes do terceiro e quarto ano do programa de cirurgia do aparelho digestivo.

Um avanço importante determinado pelo método videocirúrgico foi, sem dúvida, a redução do tempo de permanência hospitalar. Trabalhos prospectivos demonstraram metade do tempo de internação nos doentes operados por laparoscopia, quando comparado à cirurgia convencional ${ }^{43,44}$. Em séries com população de idosos, os resultados foram semeIhantes ${ }^{14,35,40,45}$. Em nosso estudo, contrariamente à literatura, ao avaliarmos o tempo de internação no pós-operatório houve semeIhança entre os dois grupos. Atribuímos este resultado à utilização de incisões menores do que as classicamente descritas, melhor analgesia e estímulo precoce à deambulação.

Acomparação da morbidade entre os métodosédifícil de ser analisada, pois diversos autores divergem na constituição dos grupos e na padronização das complicações s1, $^{45,46}$, sendo que muitas vezes comparam a colecistectomia videolaparoscópica com séries anteriores ao advento do método ${ }^{8,44,46}$. A solução seria a realização de estudos prospectivos, no entanto, a rápida aceitação do novo método tem impedido o estabelecimento desse tipo de análise ${ }^{44,45}$.

A morbidade da colecistectomia convencional foi revista recentemente com a introdução da videocirurgia. Há uma importante variação de seus valores em função da exploração da via biliar principal ${ }^{47}$, estratificação ou não de doentes idosos ${ }^{48,49}$ e porcentual de cirurgias de urgência ${ }^{49,50}$, dificultando a comparação dos dados. Quanto aos resultados da colecistectomia laparoscópica eletiva em idosos, estes situam-se entre $10 \%$ a $14,5 \%{ }^{11,12}$.

Estudos que comparam a morbidade entre os métodos apresentam taxas semelhantes $^{44,45,50}$. No entanto, o mais habitualmente relatado são publicações com menores taxas de complicações na via laparoscópica ${ }^{42,49,51,52}$. Em uma série prospectiva de 264 doentes idosos submetidos à colecistectomia pelos dois métodos, detectou-se $23,6 \%$ de complicações navia convencional contra $13,5 \%$ na laparoscópica; ressaltando ainda a maior gravidade das complicações na colecistectomia aberta ${ }^{14}$. No presente estudo, no qual estudamos idosos submetidos à colecistectomia eletiva por ambos os métodos esem outros procedimentos associados, encontramos semelhança no índice de complicação global nos grupos l e ll.
No método laparoscópico é referido um maior número de complicações cirúrgicas, destacando as lesões na realização do pneumoperitônio, perfuração da vesícula, fístulas biliares, coleções, hemorragia e iatrogenias da via biliar. Por outro lado, a videocirurgia diminuiu o número de complicações sistêmicas eas relacionadas às incisões abdominais ${ }^{12,14,51,53}$. A maior freqüência de fístulas biliares na laparoscopia é atribuída à dificuldade de dissecção da vesícula, inadequada identificação de canalículos aberrantes, lesões despercebidas da via biliar e escape da "clipagem" do ducto cístico ${ }^{36,53}$. A lesão da via biliar principal é, sem dúvida, a complicação mais temida na colecistectomia, tendo sua incidência aumentada com a laparoscopia, ocorrendo principalmente nos primeiros casos operados pelos diversos grupos ${ }^{36,37,52}$. Na análise da literatura é referido aumento de duas a seis vezes de iatrogenias sobre a via biliar, observados na fase inicial de emprego do método laparoscópico, quando comparadoà cirurgia convencional ${ }^{36}$.

Da mesma forma que o índice global de complicações, não observamos significância estatística ao analisarmos as complicações cirúrgicas dos dois grupos. Estas, quando avaliadas, quer em complicações intra como pós-operatórias, não mostraram diferenças estatisticamente significantes. Entretanto, a ocorrência de diferentes complicações nos grupos torna importante analisá-las de forma mais específica.

No grupo I, encontramos duas complicações intra-operatórias de maior gravidade, relacionadas à execução da colecistectomia: uma secção do ducto hepático comum em doente portador de vesícula escleroatrófica, reconhecida e corrigida no intra-operatório com anastomose biliodigestiva hepático jejunal em Y de Roux. Em outro doente previamente gastrectomizado, houve lesão do ramo direito da artéria hepática seguida de ligadura da mesma. As demais complicações foram tratadas com sutura das lesões. Estes doentes apresentaram evolução pós-operatória sem intercorrências. No grupo II, ocorreram lesões na execução do pneumoperitônio em três doentes. Um doente apresentou lesão de epíplon, contudo foi possível realizar a colecistectomia sem intercorrência. Em dois, houve necessidade de conversão do procedimento. Em um, por insuflação de gás carbônico no espaço pré- peritoneal e, no outro, ocorreu perfuração de intestino delgado, reparado com sutura da lesão. Todos com boa evolução pós-operatória. A análise deste grupo de complicações pode ser atribuída a uma eventual seleção de doentes no grupo l e não ao método propriamente dito. Por outro lado, as lesões relacionadas à punção do pneumoperitônio são exclusivas da videocirurgia e tendem a diminuir coma experiência adquirida.

Com relação às complicações cirúrgicas pós-operatórias, observamos também situações distintas para os dois grupos, com críticas semelhante às anteriormente descritas. No grupo I, destacaram-se as complicações relacionadas à incisão cirúrgica, ocorrendo infecção da ferida operatória em dois doentes e evisceração em três casos. Nestes, houve necessidade de ressutura da parede abdominal sendo os demais tratados de forma conservadora, evoluindo satisfatoriamente. No grupo II, observamos a migração do dreno para a cavidade abdominal em quatro doentes, com resolução através de novo procedimento videocirúrgico. Tal complicação, peculiar e infreqüente, decorreu de falha técnica na fixação do dreno, atribuída ao tipo de dreno, utilizado no início do método. Contudo, não encontramos interferência na evolução destes doentes. Ainda, como complicação apenas neste grupo, a fístula biliar ocorreu em três doentes, com drenagem precoce de secreção biliar pelo dreno abdominal e com fechamento espontâneo. Houve um caso de infecção no trocarte epigástrico, sem relevância clínica. Dois outros doentes deste grupo apresentaram má evolução pós-operatória vindo a falecer. Um decorrente de pancreatite grave no segundo dia de pós-operatório, falecendo por insuficiência de múltiplos órgãos e sistemas no terceiro dia de pós-operatório, e outro que apresentou inicialmente um $\mathrm{AVCl}$, evoluiu com broncopneumonia, coleção intra-cavitária e evicesração, havendo necessidade de intervenção cirúrgica para correção destes dois eventos. Este doente teve conversão do procedimento por dificuldade na dissecção do pedículo biliar, evidenciando os riscos habitualmente encontrados nas colecistectomias de difícil resolução.

As complicações clínicas, principalmente as pulmonares e cardiovasculares, predominam na cirurgia aberta, particularmente em idosos $^{12,51}$. Estudos utilizando a videolapa- 
RêGo REC ET AL.

roscopia ${ }^{31}$ ou comparando os dois métodos ${ }^{14}$ não observaram complicações pulmonares na via laparoscópica. Behrman et al. ${ }^{8}$, operando doentes por ambos os métodos, relataram 0 triplo de complicações cardiopulmonares na via laparotômica.

As complicações sistêmicas observadas em nosso estudo, ainda que mais freqüentes no grupo convencional, também não apresentaram diferença estatisticamente significante entre os grupos.

No grupo I, observamos complicações pulmonares e cardiovasculares. Estas foram de menor gravidade, ocorreram no pós-operatório imediato e foram tratadas com medidas clínicas de suporte. Contudo, apesar da gravidade das complicações cardiovasculares, os doentes evoluíram satisfatoriamente à terapêutica instituída.

Em apenas um doente do grupo II, já anteriormente descrito, ocorreram complicações sistêmicas, falecendo no $35^{\circ}$ dia de pós-operatório.

Em nosso estudo, os resultados demonstram baixa influência das variáveis analisadas para a ocorrência de complicações, refletindo a boa tolerância apresentada por doentes idosos quando submetidos à colecistectomia eletiva, tanto por via convencional quanto laparoscópica, ressaltando a importância de um adequado preparo no período pré-operatório.

Atualmente, a mortalidade na colecistectomia eletiva tem índice abaixo de $0,5 \%{ }^{49}$ e, nos idosos, oscila em torno de $2 \%{ }^{48,50}$. Tal diferença relaciona-se às doenças associadase ao maior número de formas complicadas da litíase biliar ${ }^{48,49,50}$, como demonstrado por Casaroli et al. ${ }^{23}$ que, no nosso serviço, analisando doentes operados por colecistite aguda, encontraram mortalidade de $20,8 \%$, demonstrando de forma inequívoca a diferença entre cirugia eletiva e de urgência nesta população.

Nos idosos, há poucos estudos que analisam especificamente a mortalidade da colecistectomia entre os dois métodos. Em um destes, os doentes foram operados eletivamente e estratificados por grupos de idade e apenas na faixa de 70 a 79 anos a laparoscopia mostrou vantagem ${ }^{5} . \mathrm{Na}$ análise de doentes com idade igual ou superior a 80 anos, encontraram mortalidade de $4,4 \%$ e I, $8 \%$, respectivamente na cirurgia aberta e na videocirurgia ${ }^{21}$, estes dados são semelhantes a outra série que encon- trou mortalidade apenas em indivíduos acima de 80 anos $^{40}$.

Em nosso estudo, contrariamente aos dados anteriormente referidos, morreram apenas doentes do grupo II, determinando uma taxa de mortalidade global de I,6\% (dois doentes), porém sem significância estatística.

Os resultados obtidos no presente estudo demonstram que o doente idoso portador de litíase vesicular sintomática e clinicamente compensado pode ser submetido à colecistectomia de forma eletiva, com baixos índices de morbimortalidade, independente da via de acesso empregada.

\section{SUMMARY}

Cholecystectomy in the elderly. EARLY RESULTS OF OPEN VERSUS LAPAROSCOPIC APPROACH

The rise of elderly population is due to the improvement of life expectancy. The biliary lithiasis is the most common condition requiring intra-abdominal surgery. Half of these patients sustain associated diseases and frequently develops acute and complicated forms of cholelithiasis. Therefore, cholecystectomy is indicated for patients with chronic symptomatic disease. During a long period open cholecystectomy has been practiced, with low complications and mortality rates. Videolaparoscopy has been recently proposed as an option in these cases, however, few studies compared its advantages over open cholecystectomy for elderly patients.

OBJECTIVE. To access the early results of the surgical treatment for cholelithiasis in the elderly submitted to an open or laparoscopic cholecystectomy.

METHODS. retrospective study with 246 patients $\geq 65$ years old, who underwent elective cholecystectomy through a subcostal incision (group I) or laparoscopic approach (group II). We employed Student's $t$ test and Spearman's correlation for statistical analysis, considering $p \leq 0,05$ as significant.

RESULTS. A hundred and twenty patients were arranged in group I and 126 in group II. Associated diseases were present in 155 cases (63). There were no differences between the groups regarding both the distribution of comorbid conditions. Conversion was evaluated in 10 patients (8.3\%). The mean operative time was 133 minutes in group I and I I 2 minutes in group II $(p=0.001)$. The mean hospital stay in group I was 3,3 days and in group II was 3.2 days $p=0.698$. Thirteen patients in each group developed complications: group I (10.8\%) and group II $(10.3 \%) p=0,896$. There was no statistis significance in complication rate related to gender, age, associated diseases and ASA when the both groups were compared. There were two deaths, both in group II, although the mortality rate was not statiscally significant between the groups $(p=0.158)$.

Conclusion. Both open and laparoscopic cholecystectomy can be practiced in the elderly with low and similar rate of complications. [Rev Assoc Med Bras 2003; 49(3): 293-9]

KEY woRDs: Cholecystectomy. Laparoscopy. Elderly complication.

\section{RefERÊNCIAS}

I. Ramos LR. A explosão demográfica da terceira idade no Brasil: uma questão de saúde pública. Gerontologia 1993; 1:3-8.

2. Organización Mundial de la Salud, OMS. La salud de las personas de edad. Ginebra: OMS; 1989. 108 p. (Série de Informes Tecnicos, 779).

3. Massie MT, Massie LB, Marrangoni AG, D'Amico FJ, Sell HW Jr. Advantages of laparoscopic cholecystectomy in the elderly and in patients with high ASA classifications. J Laparoendosc Surg 1993; 3:467-76.

4. Saxe A, Lawson J, Phillips E. Laparoscopic cholecystectomy in patients aged 65 or older. J Laparoendosc Surg 1993; 3:2 I 5-9.

5. Feldman MG, Russel JC, Lynch JT, Mattie A. Comparison of mortality rates for open and closed cholecystectomy in the elderly: Connecticut Statewide Survey. J Laparoendosc Surg 1994; 4: I65-72.

6. Miguel PR, Figueiredo FAZ, Da Rosa ALM, Reusch M, Brum SS. Colecistectomia laparoscópica no paciente idoso. Rev Col Bras Cir 1994; 21:14-6.

7. Askew AR. Surgery for gallstones in the elderly. Aust N Z J Surg 1995; 65:3 I2-5.

8. Behrman SW, Melvin WS, Babb ME, Johnson J, Ellison EC. Laparoscopic cholecystectomy in the geriatric population. Am Surg 1996; 62:386-90.

9. Firilas A, Duke Be, Max MH. Laparoscopic cholecystectomy in the elderly. Surg Endosc 1996; 10:33-5.

10. Golden WE, Cleves MA, Johnston JC. Laparoscopic cholecystectomy in the geriatric population. J Am Geriatr Soc 1996; 44: 1 380-3.

II. Magnuson TH, Ratner LE, Zenilman ME, Bender JS. Laparoscopic cholecystectomy: applicability in the geriatric population. Am Surg 1997; 1:91-5.

12. Mayol J, Martinez-Sarmiento J, Tamayo FJ, Fernández-Represa JA. Complications of 
laparoscopic cholecystectomy in the ageing patient. Age Ageing 1997; 26:77-8I.

13. Tagle FM, Laverne J, Barkin JS, Unger SW. Laparoscopic cholecystectomy in the elderly. Surg Endosc 1997; 1 1:636-8.

14. Lujan JÁ, Sanchez-Bueno F, Parrilla P, Robles R, Torralba JÁ, Gonzalez-Costea R. Laparoscopic vs. open cholecystectomy in patients aged 65 and older. Surg Laparosc Endosc 1998; 8:208-10.

15. Zenilman ME. Surgery in the elderly. Curr Probl Surg 1998; 35:99- I79.

16. CoelhoJCU, Bonilha R, Pitaki SAM, Cordeiro RMV, Salvalaggio PRO, Bonin EA et al. Prevalence of gallstones in a Brazilian population. Int Surg 1999; 84:25-8.

17. Rede Interagencial de Informações para a Saúde, RIPSA-AI4. Esperança de vida aos 65 anos de idade. Brasil. 1996. Número médio de anos de vida esperados por sobrevivente de 65 anos por sexo segundo localização. 1996; Disponível em: URL: http://www.sde.sc. gov.br/geral/qualidade.htm

18. Cervantes CastroJ, Rojas Reyna GA, Vega G, Antón J. Colecistectomía por laparoscopia en la séptima, octava, novena, y décima décadas de la vida. Cir Cir 1995; 63: 169-72.

19. González González JJ, Sanz Alvarez L, Graña López JL, Bermejo Abajo G, Navarrete Guijosa F, Martínez Rodríguez E. Litiasis biliar en pacientes mayores de 80 años. Cirurgía o tratamiento conservador? Rev Esp Enferm Dig 1997; 89:196-200.

20. Maxwell JG, Tyler BA, Maxwell BG, Brinker CC, Covington DI. Laparoscopic cholecystectomy in octogenarians. Am Surg 1998; 64:826-32.

21. Maxwell JG, Tyler BA, Rutledge R, Brinker CC, Maxwell BG, Covington DL. Cholecystectomy in patients aged 80 and older. Am J Surg 1998; 176:627-31.

22. Kahng KU, Roslyn JJ. Surgical issues for the elderly patient with hepatobiliary disease. Surg Clin North Am 1 994; 2:345-73.

23. Casaroli AA, Bevilacqua RG, Rasslan S. Análise multivariada dos fatores de risco no tratamento cirúrgico da colecistite aguda no idoso. Rev Col Bras Cir 1996; 26:1-7.

24. Montagnini AL, Jukemura J, Gianini PTH, Machado MAC, Abdo EE, PenteadoS, et al. Resultados da colecistectomia convencional. Experiência em hospital universitário. Rev Hosp Clin Fac Med São Paulo 1996; 51:93-5.

25. DuBois F, Icard P, Berthelot G, Levard H. Coelioscopic Cholecystectomy: preliminary report of 36 cases. Ann Surg 1990; 21 1 :60-2.

26. Cuschieri A, Dubois $F$, Mouiel J, Mouret $P$, Becker H, Buess $G$, et al. The European experience with laparoscopic cholecystectomy. Am J Surg 199 I; |61:385-7.

27. Szego T, Roll S, Barbosa CP, Werebe E, Soares Junior WN. Colecistectomia videolaparos- cópica: nova opção no tratamento da colecistolitíase. GED 1990; 9:27-9.

28. Macintyre IMC, Wilson RG. Laparoscopic cholecystectomy. BrJ Surg 1993; 80:552-9.

29. Deziel DJ. Complications of cholecystectomy. Incidence, clinical manifestations and diagnosis. Surg Clin North Am 1994; 4:809-23.

30. Altenfelder-Silva R. Complicações da colecistectomia vídeo laparoscópica. In: Margarido NF, Saad Junior R, Cecconello I, Martins JL, Paula RA, Soares, LA. Videocirurgia. São Paulo: Robe; 1994. p.223-43.

31. Ido K, Suzuki T, Kimura K, Taniguchi Y, Kawamoto C, Isoda N, et al.. Laparoscopic cholecystectomy in the elderly: analysis of preoperative risk factors and postoperative complications. J Gastroenterol Hepatol I 995; 10:517-22.

32. Pasterna KR. Preanesthesia evaluation of surgical patient. In: The American Society of Anesthesiologists-ASA. Pennsylvania: Lippincott-Raven; 1996. v.24, p.206-19.

33. Böckler D, Geoghegan J, Klein M, WeibMann $\mathrm{Q}$, Turan M, Meyer L, et al. Implications of laparoscopic cholecystectomy for surgical residency training. J Soc Laparoendosc Surg 1999; 3:19-22.

34. Rêgo REC, Casaroli AA, Silva R, Pacheco Júnior AM, Fava J. Morbidade e mortalidade do tratamento eletivo da litíase biliar no idoso. Rev Col Bras Cir 1995; 22(Supl 2): 102. [Resumo]

35. Elli M, Poliziani D, Colli Franzoni P, Vignati GA, Taschieri AM. Impact of the introduction of laparoscopic cholecystectomy on the population aged 70 and over. Hepatogastroenterology 2001; 48: 1295-7.

36. Deziel DJ, Millikan KW, Economou SG, Doolas A, Ko S-T, Airan MC. Complications of laparoscopic cholecystectomy: a national survey of 4,292 hospitals and an analysis of 77,604 cases. Am J Surg 1993; 165:9-14.

37. Vecchio R, Macfadyen Bv, Latteri S. Laparoscopic cholecystectomy: an analisys on I I 4,005 cases of United States series. Int Surg 1998; 83:215-9

38. CoelhoJCU, Vianna R, Aranha A, Sobreiro BP, Borges AM. Tipo, número e tamanho de cálculos da vesícula biliar. Estudo prospectivo de 300 casos de colelitíase. Rev Col Bras Cir 1999; 26:265-8

39. Hawasli A, Brown E. The effect of drains in laparoscopic cholecystectomy. J Laparoendosc Surg 1994; 4:393-8.

40. Brunt LM, Quasebarth MA, Dunnegan DL, Soper NJ. Outcomes analysis of laparoscopic cholecystectomy in the extremely elderly. Surg Endosc 200I; 15:700-5

41. Buanes T, Mjåland O. Complications in laparoscopic and open cholecystectomy: a prospective comparative trial. Surg Laparosc Endosc 1996; 6:266-72.

42. Traverso LW, Koo KP, Hargrave K, Unger SW, Roush TS, Swanstrom LL, et al. Standardizing laparoscopic procedure time and determining the effect of patient age/gender and presence or absence of surgical residents during operation: a prospective multicenter trial. Surg Endosc 1997; 1 1:226-9.

43. Trondsen E, Reiertsen O, Andersen OK, Kjærsgaard P. Laparoscopic and open cholecystectomy: a prospective, randomized study. Eur J Surg 1993; I59:217-21.

44. Porte RJ, De Vries BC. Laparoscopic versus open cholecystectomy: a prospective matched-cohort study. HPB Surg 1996; 9:7I-5.

45. Huang SM, Wu CW, Lui WY, P'eng FK. A prospective randomized study of laparoscopic $v$. open cholecystectomy in aged patients with cholecystolithiasis. S Afr J Surg 1996; 34: 17780.

46. Jatzko GR, Lisborg PH, Pertl AM, Stettner HM. Multivariate comparison of complications after laparoscopic cholecystectomy and open cholecystectomy. Ann Surg 1995; 22 1:38 I-6.

47. Clavien PA, Sanabria JR, Mentha G, Borst F, Buhler L, Roche B, et al. Recent results of elective open cholecystectomy in a North American and a European center: comparison of complications and risk factors. Ann Surg 1992; 216:618-26.

48. Morgenstern L, Wong L, Berci G. Twelve hundred open cholecystectomies before the laparoscopic era. A standard for comparison. Arch Surg 1992; 127:400-3.

49. Roslyn JJ, Binns GS, Hughes EFX, SaundersKirkwood K, Zinner MJ, Cates JA. Open clolecystectomy: a contemporary analysis of 42,474 patients. Ann Surg 1993; 21 8: 129-37.

50. Escarce J], Shea JÁ, Chen W, Qian Z, Schwartz JS. Outcomes of open cholecystectomy in the elderly: a longitudinal analysis of $2 \mathrm{I}, 000$ cases in the prelaparoscopic era. Surgery 1995; II7:156-64.

51. Fisichella PM, Di Stefano A, Di Carlo I, La Greca G, Russello D, Latteri F. Efficacy and safety of elective laparoscopic cholecystectomy in elderly: a case-controlled comparison with the open approach. Ann Ital Chir 2002; 73: 149-54.

52. Shea JÁ, Healey MJ, Berlin JÁ, Clarke JR, Malet PF, Staroscik RN, et al. Mortality and complications associated with laparoscopic cholecystectomy: a meta-analysis. Ann Surg 1996; 224:609-20.

53. Hannan EL, Imperato PJ, Nenner RP, Starr H. Laparoscopic and open cholecystectomy in New York State: mortality, complication, and choice of procedure. Surgery 1999; |25:223-3|.

Artigo recebido: 13/09/02 Aceito para publicação:06/03/03 